\title{
DDIT3 Expression in Liposarcoma Development
}

\author{
Christina Kåbjörn Gustafsson, ${ }^{1}$ Katarina Engström, ${ }^{2}$ and Pierre Åman ${ }^{1}$ \\ ${ }^{1}$ Sahlgrenska Cancer Center, Department of Pathology, Institute of Biomedicine, University of Gothenburg, \\ P.O. Box 425, 40530 Gothenburg, Sweden \\ ${ }^{2}$ Department of Oncology, Institute of Medical Sciences, University of Gothenburg, Gothenburg, Sweden
}

Correspondence should be addressed to Pierre Åman; pierre.aman@gu.se

Received 5 December 2013; Accepted 13 February 2014; Published 25 March 2014

Academic Editor: Enrique de Alava

Copyright (C) 2014 Christina Kåbjörn Gustafsson et al. This is an open access article distributed under the Creative Commons Attribution License, which permits unrestricted use, distribution, and reproduction in any medium, provided the original work is properly cited.

\begin{abstract}
Liposarcomas are mesenchymal tumors containing variable numbers of lipoblasts or adipocytes. The most common entities, well differentiated/dedifferentiated liposarcoma (WDLS/DDLS) and myxoid/round cell liposarcoma (MLS/RCLS), are both characterized by genetic rearrangements that affect the expression of the transcription factor DDIT3. DDIT3 induces liposarcoma morphology when ectopically expressed in a human fibrosarcoma. The role of DDIT3 in lipomatous tumors is, however, unclear. We have analyzed the expression of DDIT3 in 37 cases of liposarcoma (WDLS/DDLS $n=10$, MLS/RCLS $n=16$, and pleomorphic liposarcomas (PLS) $n=11$ ) and 11 cases of common benign lipomas. Major cell subpopulations of WDLS/DDLS and MLS/RCLS tumors were found to express DDIT3 or the derived fusion protein, whereas PLS cases showed only a few positive cells. The lipomas contained large subpopulations expressing DDIT3. No correlation between numbers of DDIT3 expressing cells and numbers of lipoblasts/adipocytes was found. In vitro adipogenic treatment of two DDIT3 expressing cell lines induced lipid accumulation in small subpopulations only. Our results suggest a dual, promoting and limiting, role for DDIT3 in the formation of lipoblasts and liposarcoma morphology.
\end{abstract}

\section{Introduction}

Adipocytic tumors are the most frequent types of soft tissue tumors occurring in humans. They also represent the largest single group of mesenchymal tumors [1] and are characterized by the more or less prominent presence of lipoblasts or adipocytes. Some of the adipocytic neoplasms are characterized by recurrent tumor type specific genetic rearrangements. Most WDLS/DDLS cases contain amplified segments of chromosome 12q13-15 carried as ring chromosomes or large marker chromosomes [2-4]. The amplified regions contain many tumor associated genes and among them DDIT3. MLS/RCLS carry a rearranged DDIT3 fused to FUS or EWSR1 [5, 6]. DDIT3 is expressed and involved in the regulation of adipocyte development and we have previously shown that expression of DDIT3 in a low differentiated fibrosarcoma cell line results in morphological conversion towards a liposarcoma phenotype [7]. These observations suggest that expression of DDIT3 protein could be a common phenotype determining factor for several types of lipomatous tumors. The aim of the present study was to test this hypothesis by investigating the expression of the DDIT3 protein in 3 different subtypes of liposarcoma and in common lipoma.

We further evaluated the role of DDIT3 expression by studying lipoblast formation in cultured liposarcoma cells treated with adipogenic factors. DDIT3 expression is tightly regulated at several levels including translation and protein degradation [8]. This makes expression analysis at protein level the most relevant approach.

\section{Material and Methods}

2.1. Immunohistochemistry. Paraffin embedded sections were obtained from our pathology department in conformity with Swedish legislation. The material consists of 11 lipomas, 11 PLS, 10 WDLS, and 16 MLS/RCLS.

Sections were prepared from routine paraffin embedded tumor tissue samples from 48 cases of lipomas and liposarcomas (Table 1). Immunohistochemistry (IHC) was performed 
TABLE 1: Cases and DDIT3 expression.

\begin{tabular}{|c|c|c|c|c|c|c|c|}
\hline \multirow{2}{*}{ Case } & \multirow{2}{*}{ Age } & \multirow{2}{*}{\multicolumn{2}{|c|}{ Site }} & \multicolumn{2}{|c|}{ DDIT3 \% } & \multirow{2}{*}{ FUS/DDIT3 rearrangement } & \multirow{2}{*}{ Histological diagnosis } \\
\hline & & & & Nucleus & Cytoplasm & & \\
\hline 1 & 72 & im & Axilla & 79 & 0 & ND & WLDS \\
\hline 2 & 69 & $\mathrm{im}$ & Thigh & 34 & 0 & ND & WLDS \\
\hline 3 & 77 & im & Hip & 82 & 0 & $\mathrm{ND}$ & WLDS \\
\hline 4 & 71 & other & Retroperitoneal & 0 & 36 & ND & DDLS \\
\hline 5 & 42 & other & Abdomen & 70 & 0 & ND & DDLS \\
\hline 6 & 80 & other & Abdomen & 94 & 0 & ND & DDLS \\
\hline 7 & 73 & other & Inguinal & 40 & 0 & ND & WDLS \\
\hline 8 & 77 & other & Peritoneal & 84 & 0 & $\mathrm{ND}$ & WDLS \\
\hline 9 & 60 & other & Inguinal & 69 & 0 & ND & WDLS \\
\hline 10 & 67 & im & Thigh & 81 & 0 & ND & WDLS/DDLS \\
\hline 11 & 80 & im & Thigh & 59 & 0 & Yes & MLS \\
\hline 12 & 34 & $\mathrm{im}$ & Thigh & 61 & 0 & Yes & MLS \\
\hline 13 & 49 & $\mathrm{im}$ & Hip & 46 & 0 & Yes & MLS \\
\hline 14 & 46 & $\mathrm{im} / \mathrm{sc}$ & Hip/thigh & 71 & 0 & Yes & MLS \\
\hline 15 & 39 & im & Thigh & 24 & 0 & Yes & MLS \\
\hline 16 & 17 & $\mathrm{im}$ & Thigh & 39 & 0 & $\mathrm{Nev}$ & MLS \\
\hline 17 & 45 & $\mathrm{im}$ & Thigh & 58 & 0 & Yes & MLS \\
\hline 18 & 76 & $\mathrm{sc}$ & Back & 44 & 0 & Yes & MLS/RCLS \\
\hline 19 & 42 & im & Abdomen & 70 & 0 & Yes & MLS/RCLS \\
\hline 20 & 33 & other & Abdomen & 50 & 0 & Yes & MLS/RCLS \\
\hline 21 & 45 & other & Abdomen & 41 & 0 & $\mathrm{Nev}$ & MLS/RCLS \\
\hline 22 & 73 & im & Leg & 49 & 0 & $\mathrm{Nev}$ & MLS/RCLS \\
\hline 23 & 38 & im & Thigh & 64 & 0 & Yes & MLS/RCLS \\
\hline 24 & 36 & $\mathrm{im}$ & Thigh & 73 & 0 & Yes & MLS/RCLS \\
\hline 25 & 37 & im & Thigh & 50 & 0 & Yes & MLS/RCLS \\
\hline 26 & 46 & $\mathrm{im}$ & Hip & 0 & 57 & Yes & RCLS \\
\hline 27 & 84 & im & Arm & 0 & 9 & ND & PLS \\
\hline 28 & 57 & im & Iliopsoas & 0 & 0 & ND & PLS \\
\hline 29 & 89 & im & Leg & 9 & 0 & ND & PLS \\
\hline 30 & 88 & $\mathrm{im}$ & Thigh & 2 & 0 & ND & PLS \\
\hline 31 & 70 & $\mathrm{im}$ & Thigh & 18 & 0 & ND & PLS \\
\hline 32 & 72 & im & Neck & 0 & 7 & $\mathrm{ND}$ & PLS \\
\hline 33 & 81 & $\mathrm{im}$ & Axilla & 0 & 6 & ND & PLS \\
\hline 34 & 69 & im & Thigh & 0 & 1 & ND & PLS \\
\hline 35 & 61 & $\mathrm{im}$ & Thigh & 0 & 9 & ND & PLS \\
\hline 36 & 51 & im & Hip & 0 & 0 & ND & PLS \\
\hline 37 & 54 & $\mathrm{im}$ & Hip & 0 & 20 & ND & PLS \\
\hline 38 & 44 & $\mathrm{sc}$ & Inguinal & 53 & 0 & ND & Lipoma \\
\hline 39 & 44 & $\mathrm{sc}$ & Shoulder & 54 & 0 & ND & Lipoma \\
\hline 40 & 51 & $\mathrm{sc}$ & Shoulder & 68 & 0 & ND & Lipoma \\
\hline 41 & 56 & sc & Neck & 32 & 0 & ND & Lipoma \\
\hline 42 & 40 & $\mathrm{sc}$ & Hip & 40 & 0 & ND & Lipoma \\
\hline 43 & 41 & sc & Neck & 15 & 0 & ND & Lipoma \\
\hline 44 & 72 & sc & Thigh & 38 & 0 & ND & Lipoma \\
\hline 45 & 38 & sc & Arm & 73 & 0 & ND & Lipoma \\
\hline 46 & 66 & $\mathrm{sc}$ & Arm & 71 & 0 & ND & Lipoma \\
\hline 47 & 66 & $\mathrm{sc}$ & Thigh & 81 & 0 & ND & Lipoma \\
\hline 48 & 47 & sc & Back & 75 & 0 & $\mathrm{ND}$ & Lipoma \\
\hline
\end{tabular}

Clinical, diagnostic, and DDIT expression data on the investigated cases. DDIT3 expression is shown as percent positive cells. Abbreviations: im: 0 intramuscular; sc: subcutaneous; ND: not analyzed; nev: not possible to evaluate. 


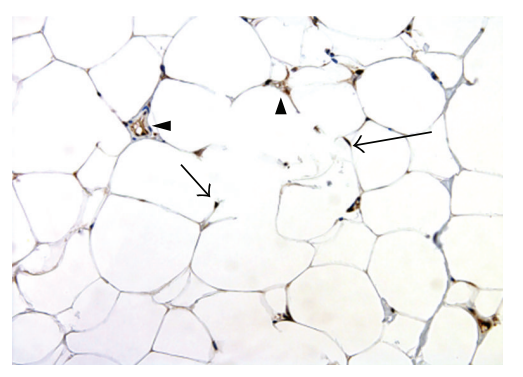

(a)

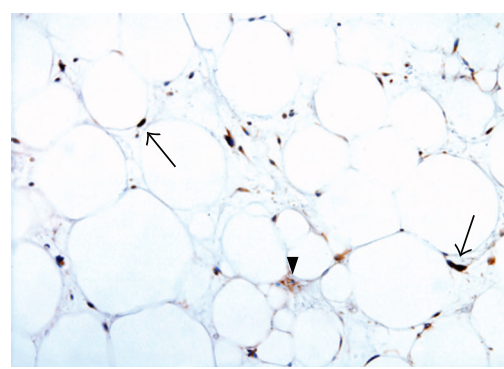

(b)

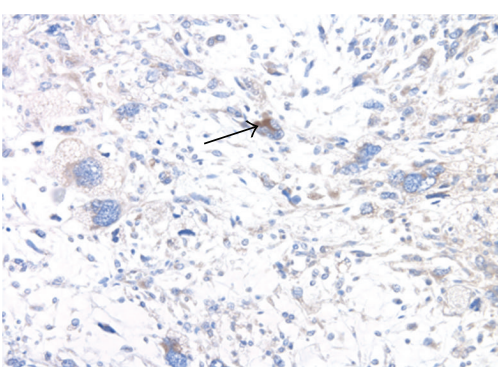

(c)

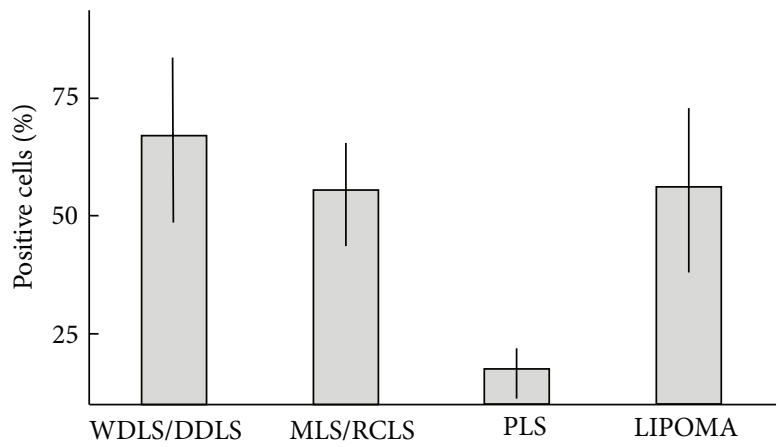

(d)

FIGURE 1: DDIT3 immunohistochemistry analysis of lipomatous tumors. (a) Lipoma, nuclear, and DDIT3 expression (arrow) and capillaries are indicated by arrowheads. (b) WDLS/DDLS, nuclear, and cytoplasmic DDIT3 expression (arrow). (c) PLS, nuclear, and cytoplasmic DDIT3 expression in lipoblasts (arrow). (d) Mean value and standard deviation of percent positively stained cells in 10 cases of WDLS/DDLS, 16 cases of MLS/RCLS, 11 cases of PLS, and 11 cases of lipoma.

as described previously [7] using the DDIT3 specific antibody Gadd153 R20 from Santa Cruz Biotechnology at a dilution of $1: 200$.

The histological specimens were examined and evaluated in a blinded fashion by two examiners. The proportion of stained tumor cells was counted at 200x magnification. Cells with nuclear and cytoplasmic expression were counted avoiding inflammatory cells, endothelial cells, and necrotic areas. Three different areas in each slide were counted and a mean value was calculated.

2.2. Fluorescence In Situ Hybridization Analysis. Interphase FISH analysis of formalin-fixed tumor tissue was performed on $1-4 \mu \mathrm{m}$ paraffin sections. Three break-apart probes, DDIT3, FUS, and EWSR1 (Vysis, Inc., Downers Grove, IL), were used according to protocols supplied by the manufacturer. Nuclei were counterstained with $10 \mu \mathrm{L}$ $4^{\prime}, 6^{\prime}$,-diamidino- $2^{\prime}$-phenylindole dihydrochloride (DAPI). The sections were analyzed and reanalyzed by two independent reviewers. At least 100 nuclei per section were scored. The interpretation of intact, fusion, and split signals was based on guidelines recommended by the manufacturer and from other clinical laboratories using this method.

2.3. In Vitro Adipogenesis. The GOT3 cell line, established from a WDLS tumor [4], was used to study adipogenic differentiation. The cells were cultured in RPMI 1640 until $100 \%$ confluence, after which the medium was changed to adipogenesis induction medium (PT3004 containing human recombinant insulin, dexamethasone, indomethacin, and 3isobutyl-1-methylxanthine (IBMX); Cambrex, East Rutherford, NJ) or maintenance medium (MM; RPMI 1640 containing $8 \%$ fetal calf serum). The cells were treated with adipogenesis induction medium for 3 days, followed by 1 to 3 days in MM. This was repeated three times. Control cultures were fed with only MM following the same schedule. After completed cycles, the cells were cultured for 7 more days in MM with replacement of the medium every 2 to 3 days. The cells were inspected using a microscope, and accumulation of fat was assessed by staining the cells with Oil Red $\mathrm{O}$ after fixation with $4 \%$ buffered formalin.

\section{Results and Discussion}

Clinical data, histological features, and DDIT3 protein expression are detailed in Table 1 and representative examples of IHC staining are shown in Figure 1. DDIT3 expression was detected in all but 2 of the 48 investigated cases.

In WDLS/DDLS tumors $40-94 \%$ of the cells expressed DDIT3 (Table 1). The constitutive DDIT3 expression in this tumor type could be explained by recurrent gene amplicons that carry the DDIT3 in this tumor type $[2,4]$. There was no obvious difference in numbers of DDIT3 expressing cells between WDLS and DDLS in this small series of tumors.

In MLS/RCLS, DDIT3 is expressed as part of the FUSDDIT3 or EWSR1-DDIT3 fusion oncoproteins [5, 9]. Transcription of the fusion oncogenes is regulated by the ubiquitously active promoters of the FUS or EWSR1 partner genes 

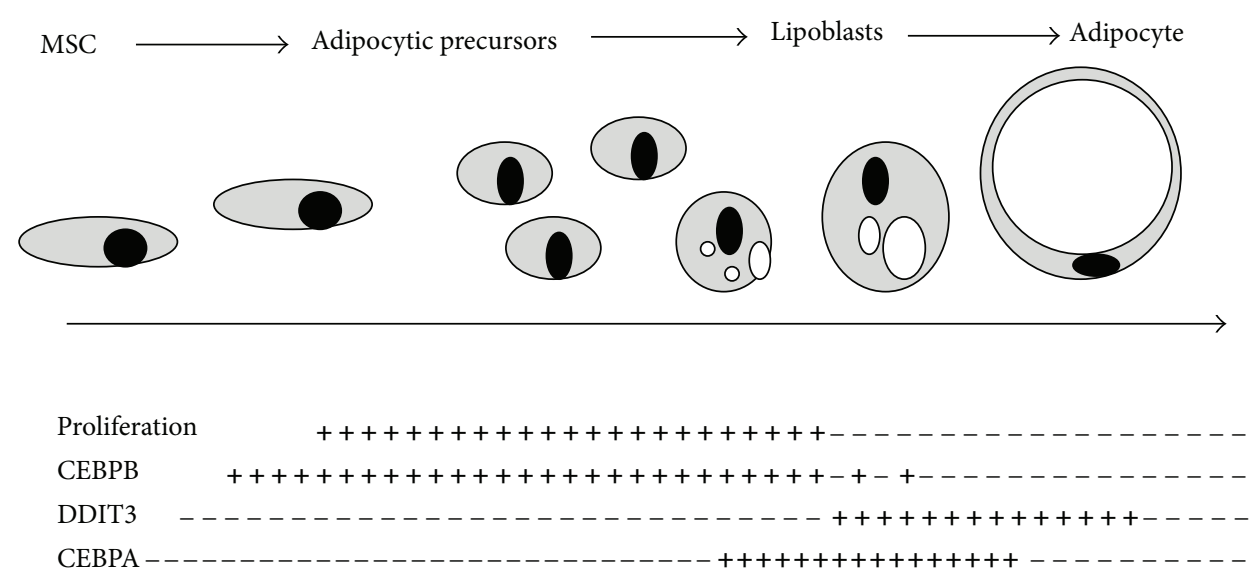

(a)

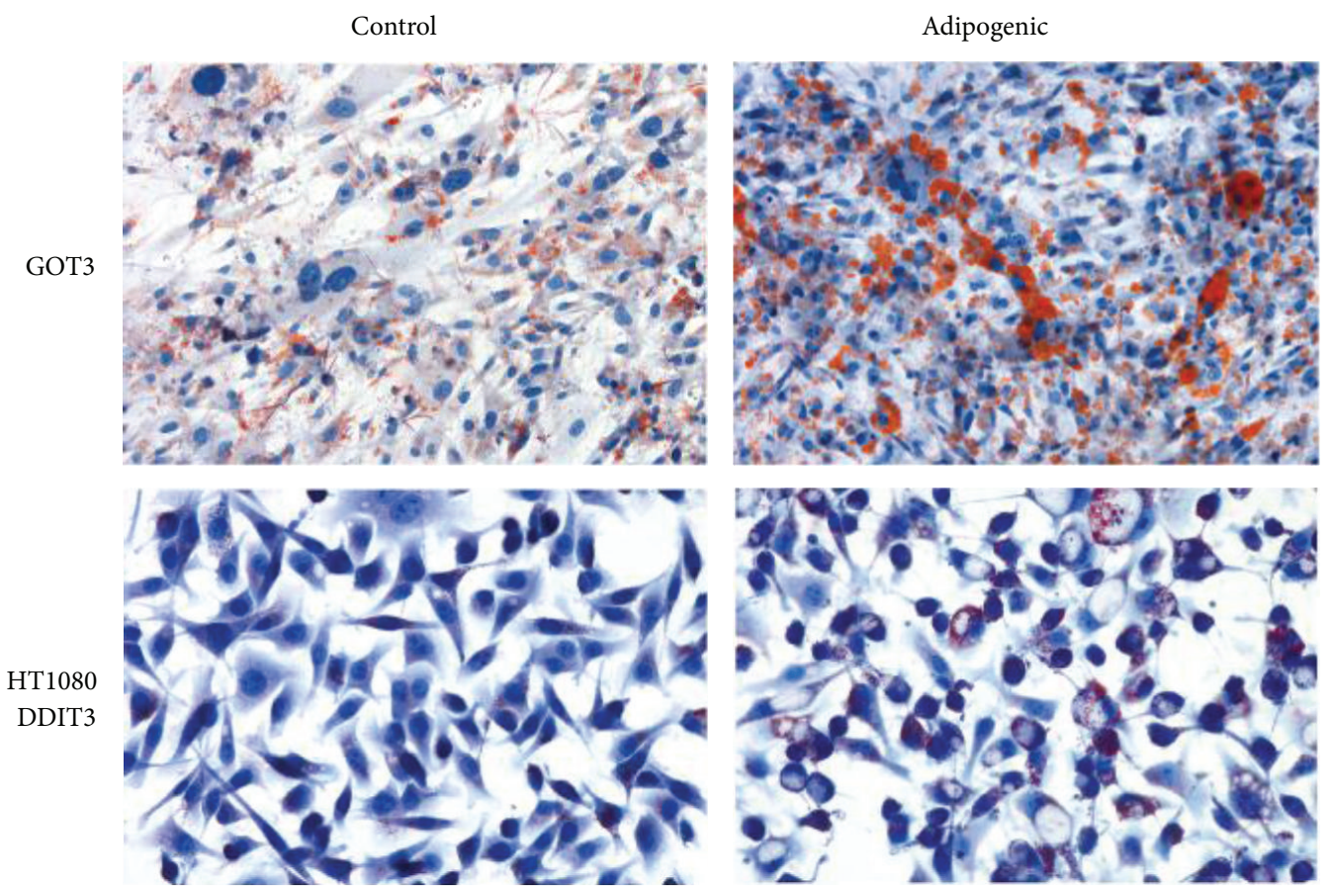

(b)

FIGURE 2: Adipocytic differentiation. (a) Schematic presentation of CEBPB, CEBPA, and DDIT3 expression in adipocyte differentiation. (b) Lipoblast formation and accumulation of lipids in GOT3 WDLS/DDLS derived cell line (top panels) and DDIT3 transfected HT1080 fibrosarcoma cell line (bottom panels) cultured in control or adipogenic medium. Oil Red O staining shows lipids in red.

[6]. The MLS/RCLS tumors contained 24-73\% FUS-DDIT3 or EWSR1-DDIT3 positive cells (Table 1). The fact that the fusion proteins could be detected only in a subpopulation of the tumor cells indicates that the levels of fusion proteins are regulated also at posttranscriptional levels. There was no clear difference in numbers of positive cells between typical myxoid cases and those with round cell components.

A cytoplasmic DDIT3 staining pattern was seen in most PLS cases but only in a low percentage of the cells. Three tumors (cases 29, 30, and 31) showed a nuclear staining pattern of DDIT3 in a minority of the cells, mostly with bizarre nuclei. There are no reported recurrent genetic aberrations causing DDIT3 expression in PLS. Instead, stress induced expression may explain the presence of DDIT3 protein in these tumors. Cytoplasmic or nuclear DDIT3 expression may be induced as a response to various stress conditions such as DNA damage, hypoxia, and lack of nutrients [10-12]. Such conditions are common in tumor tissues and may thus lead to DDIT3 expression.

The highly differentiated common lipoma expressed DDIT3 in $15-81 \%$ of the cells (Figure 1). The DDIT3 protein was found both in univacuolated fat cells and surrounding spindle cells. DDIT3 is not amplified or rearranged in lipomas. Instead, most of the cases carry a rearranged HMGA2 
gene that may promote adipocytic differentiation [13]. The DDIT3 expression found in lipoma cells may therefore result from a terminal adipocyte differentiation program in these tumor cells [14]. Thus, there is currently no reason to believe that aberrant DDIT3 expression is involved in the development of this tumor type.

DDIT3 is normally expressed late in adipocyte differentiation together with the related transcription factor CEBPA [14]. The timing of expression of DDIT3 and CEBP factors is crucial for normal differentiation (Figure 2). Premature or overexpression of DDIT3 in preadipocytes may block terminal differentiation [15-17]. In the context of liposarcoma development, aberrant DDIT3 or FUS-DDIT3 expression in primitive tumor cells may open an adipocytic differentiation pathway but block later stages of adipocyte development. We speculate that only a small minority of the tumor cells would make it through the block and differentiate to lipoblasts. This would also explain the lack of correlation between numbers of DDIT3 expressing cells and numbers of lipoblasts in the investigated tumors.

To test this hypothesis, the WDLS/DDLS derived cell line GOT3 was analyzed after adipogenic treatment. This cell line carries a large chromosome 12 derived amplicon including the DDIT3 gene and was found to express DDIT3 constitutively in almost all cells [4]. A limited accumulation of lipids in sporadic cells was seen under standard culture (Figure 2(b)). Transfer to adipogenic culture conditions resulted in lipid accumulation and lipoblast development but only in a minority of the cells. Similar results were obtained for a fibrosarcoma cell line stably transfected with EGFP tagged DDIT3 (Figure 2(b)). These results suggest that aberrant expression of DDIT3 can promote a liposarcoma phenotype in human primitive sarcoma cells [7].

FUS-DDIT3 transfected mouse mesenchymal stem cells cause MLS/RCLS like tumors when injected in mice and FUSDDIT3 transfection transforms 3T3 mouse fibroblasts $[18,19]$. This shows that FUS-DDIT3 is a powerful oncogene. The FUS-DDIT3 protein maintains the capacity of DDIT3 to form heterodimers with CEBPA and CEBPB and has been shown to modify or block the activity of its dimer partners $[17,19]$. Blocking of CEBPA could inhibit terminal differentiation [17]. Since transcription of the fusion gene is driven by the ubiquitously active FUS promoter, failed expression timing of this abnormal variant of DDIT3 may also contribute to the oncogenic activity. The FUS part of FUS-DDIT3 was found to be necessary for transformation of 3T3 fibroblasts [19] and forced expression of FUS-DDIT3 failed to induce cell cycle arrest as reported for DDIT3.

In contrast to FUS-DDIT3, forced expression of the DDIT3 protein in mesenchymal cells or in transgenic mice gave no evidence of transformation or tumorigenic activity [20]. The normal DDIT3 protein thus cannot be considered a driving oncoprotein. DDIT3 expression may, however, halt proliferation $[19,21]$. In WLDLS and DDLS it is overexpressed together with several other proto-oncogenes such as MDM2 and CDK4 that together may cause tumor development. Aberrantly expressed, DDIT3 may in this context act as a promoting or/and tumor-type directing factor by blocking or interfering with the adipocyte differentiation program and the associated growth termination.

Originally described as a nuclear DNA-binding transcription factor, stress induced DDIT3 has later been reported to localize in the cytoplasmic compartment [21]. Cytoplasmic DDIT3 was shown to induce partially distinct effects compared to nuclear DDIT3. One proposed mechanism was sequestration of its CEBP family dimerization partners [21]. In the present study we observed cytoplasmic DDIT3 primarily in PLS cells containing bizarre nuclei, thus supporting a stress related mechanism behind the expression in these cells. Stress induced DDIT3 expression is more likely temporary but could anyway trigger some of the low differentiated PLS cells into an adipocytic differentiation path explaining the presence of lipoblasts in this tumor type. As in WLDLS and DDLS, expression of DDIT3 may also contribute to tumor development by halting the adipocytic program and associated growth termination.

In summary, DDIT3 is expressed in subpopulations of tumor cells in all 4 investigated lipomatous tumor types. There was no obvious difference between the number of DDIT3 expressing cells in the more aggressive DDLS and RCLS compared to WDLS and MLS. Furthermore, DDIT3 was expressed at comparable levels in benign lipomas. Only a minority of DDIT3 expressing sarcoma cells responded to adipogenic conditions in vitro indicating a complex role for DDIT3 as a phenotype directing factor in lipomatous tumors.

\section{Conflict of Interests}

The authors declare that there is no conflict of interests regarding the publication of this paper.

\section{Acknowledgments}

This work was supported by grants from the Swedish Cancer Society, Assar Gabrielsson Research Foundation, Johan Jansson Foundation for Cancer Research, Socialstyrelsen, Swedish Society for Medical Research, Swedish Children's Cancer Society, BioCARE National Strategic Research Program at the University of Gothenburg, and Wilhelm and Martina Lundgren Foundation for Scientific Research.

\section{References}

[1] C. D. M. Fletcher, K. Krishnan Unni, and F. Mertens, Tumors of Soft Tissue and Bone, IARC Press, 2000.

[2] F. Pedeutour, A. Forus, J.M. Berner et al., "Structure of the supernumerary ring and giant rod chromosomes in adipose tissue tumors," Genes, Chromosomes \& Cancer, vol. 24, no. 1, pp. 30-41, 1999.

[3] F. Pedeutour, R. F. Suijkerbuijk, A. Forus et al., "Complex composition and co-amplification of SAS and MDM2 in ring and giant rod marker chromosomes in well-differentiated liposarcoma," Genes, Chromosomes \& Cancer, vol. 10, no. 2, pp. 85-94, 1994.

[4] F. Persson, A. Olofsson, H. Sjögren et al., "Characterization of the $12 \mathrm{q}$ amplicons by high-resolution, oligonucleotide array 
CGH and expression analyses of a novel liposarcoma cell line," Cancer Letters, vol. 260, no. 1-2, pp. 37-47, 2008.

[5] A. Crozat, P. Aman, N. Mandahl, and D. Ron, "Fusion of $\mathrm{CHOP}$ to a novel RNA-binding protein in human myxoid liposarcoma," Nature, vol. 363, no. 6430, pp. 640-644, 1993.

[6] P. Åman, I. Panagopoulos, C. Lassen et al., "Expression patterns of the human sarcoma-associated genes FUS and EWS and the genomic structure of FUS," Genomics, vol. 37, no. 1, pp. 1-8, 1996.

[7] K. Engström, H. Willén, C. Kåbjörn-Gustafsson et al., “The myxoid/round cell liposarcoma fusion oncogene FUS-DDIT3 and the normal DDIT3 induce a liposarcoma phenotype in transfected human fibrosarcoma cells," American Journal of Pathology, vol. 168, no. 5, pp. 1642-1653, 2006.

[8] C. Jousse, A. Bruhat, V. Carraro et al., "Inhibition of CHOP translation by a peptide encoded by an open reading frame localized in the chop 5'UTR," Nucleic Acids Research, vol. 29, no. 21, pp. 4341-4351, 2001.

[9] I. Panagopoulos, M. Höglund, F. Mertens, N. Mandahl, F. Mitelman, and P. Åman, "Fusion of the EWS and CHOP genes in myxoid liposarcoma," Oncogene, vol. 12, no. 3, pp. 489-494, 1996.

[10] A. Benavides, D. Pastor, P. Santos, P. Tranque, and S. Calvo, "CHOP plays a pivotal role in the astrocyte death induced by oxygen and glucose deprivation," Glia, vol. 52 , no. 4 , pp. 261275, 2005.

[11] Y. Ma, J. W. Brewer, J. Alan Diehl, and L. M. Hendershot, “Two distinct stress signaling pathways converge upon the CHOP promoter during the mammalian unfolded protein response," Journal of Molecular Biology, vol. 318, no. 5, pp. 1351-1365, 2002.

[12] A. A. Welihinda, W. Tirasophon, and R. J. Kaufman, "The cellular response to protein misfolding in the endoplasmic reticulum," Gene Expression, vol. 7, no. 4-6, pp. 293-300, 1999.

[13] E. F. P. M. Schoenmakers, S. Wanschura, R. Mols, J. Bullerdiek, H. van den Berghe, and W. J. M. van de Ven, "Recurrent rearrangements in the high mobility group protein gene, HMGI-C, in benign mesenchymal tumours," Nature Genetics, vol. 10, no. 4, pp. 436-444, 1995.

[14] G. J. Darlington, S. E. Ross, and O. A. MacDougald, "The role of C/EBP genes in adipocyte differentiation," The Journal of Biological Chemistry, vol. 273, no. 46, pp. 30057-30060, 1998.

[15] G. Adelmant, J. D. Gilbert, and S. O. Freytag, "Human translocation liposarcoma-CCAAT/enhancer binding protein (C/EBP) homologous protein (TLS-CHOP) oncoprotein prevents adipocyte differentiation by directly interfering with C/EBP $\beta$ function," The Journal of Biological Chemistry, vol. 273, no. 25, pp. 15574-15581, 1998.

[16] N. Batchvarova, X.-Z. Wang, and D. Ron, "Inhibition of adipogenesis by the stress-induced protein CHOP (Gadd153)," EMBO Journal, vol. 14, no. 19, pp. 4654-4661, 1995.

[17] M. Kuroda, T. Ishida, M. Takanashi, M. Satoh, R. Machinami, and T. Watanabe, "Oncogenic transformation and inhibition of adipocytic conversion of preadipocytes by TLS/FUS-CHOP type II chimeric protein," American Journal of Pathology, vol. 151, no. 3, pp. 735-744, 1997.

[18] N. Riggi, L. Cironi, P. Provero et al., "Expression of the FUSCHOP fusion protein in primary mesenchymal progenitor cells gives rise to a model of myxoid liposarcoma," Cancer Research, vol. 66, no. 14, pp. 7016-7023, 2006.

[19] H. Zinszner, R. Albalat, and D. Ron, "A novel effector domain from the RNA-binding protein TLS or EWS is required for oncogenic transformation by CHOP," Genes and Development, vol. 8, no. 21, pp. 2513-2526, 1994.
[20] J. Pérez-Losada, M. Sánchez-Martín, M. A. Rodríguez-García et al., "Liposarcoma initiated by FUS/TLS-CHOP: the FUS/TLS domain plays a critical role in the pathogenesis of liposarcoma," Oncogene, vol. 19, no. 52, pp. 6015-6022, 2000.

[21] A. Jauhiainen, C. Thomsen, L. Strömbom et al., "Distinct cytoplasmic and nuclear functions of the stress induced protein DDIT3/CHOP/GADD153," PLoS ONE, vol. 7, no. 4, Article ID e33208, 2012. 


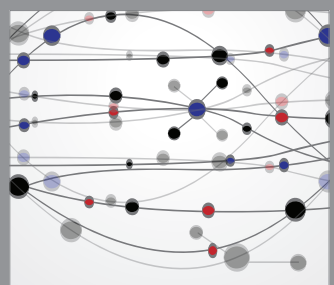

The Scientific World Journal
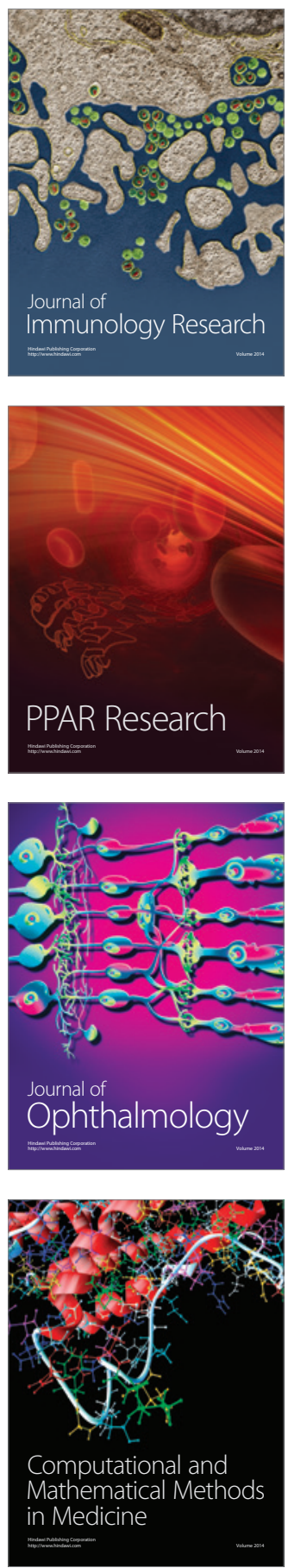

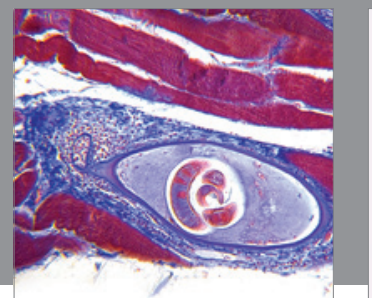

Gastroenterology

Research and Practice
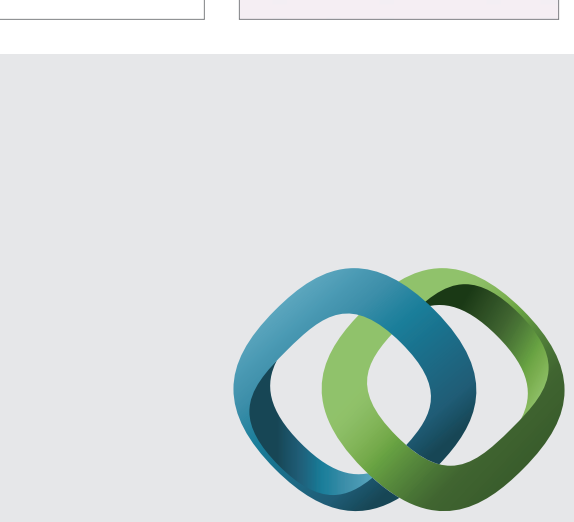

\section{Hindawi}

Submit your manuscripts at

http://www.hindawi.com
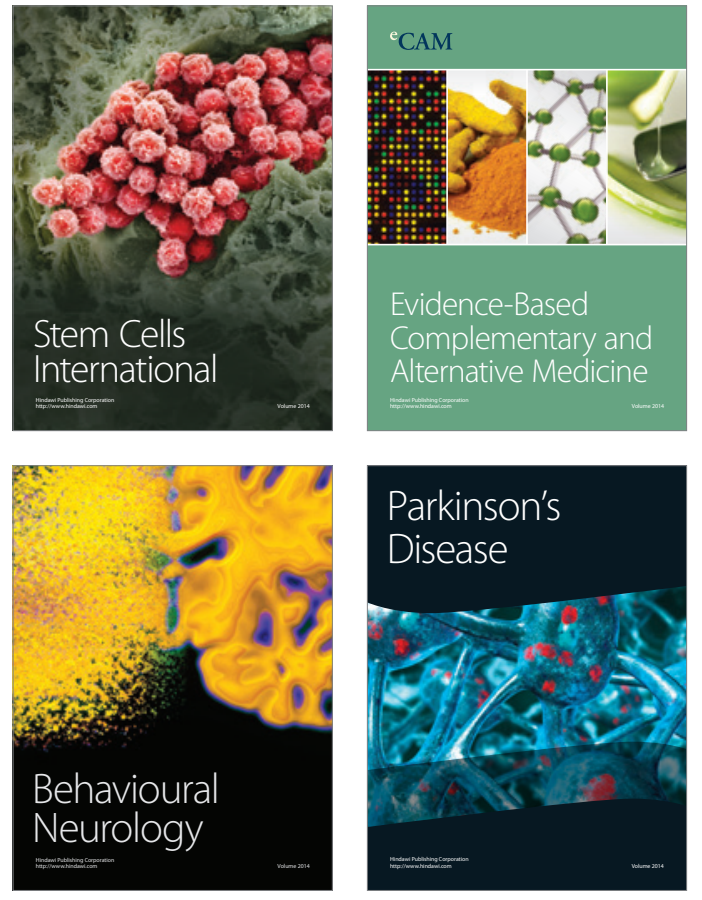
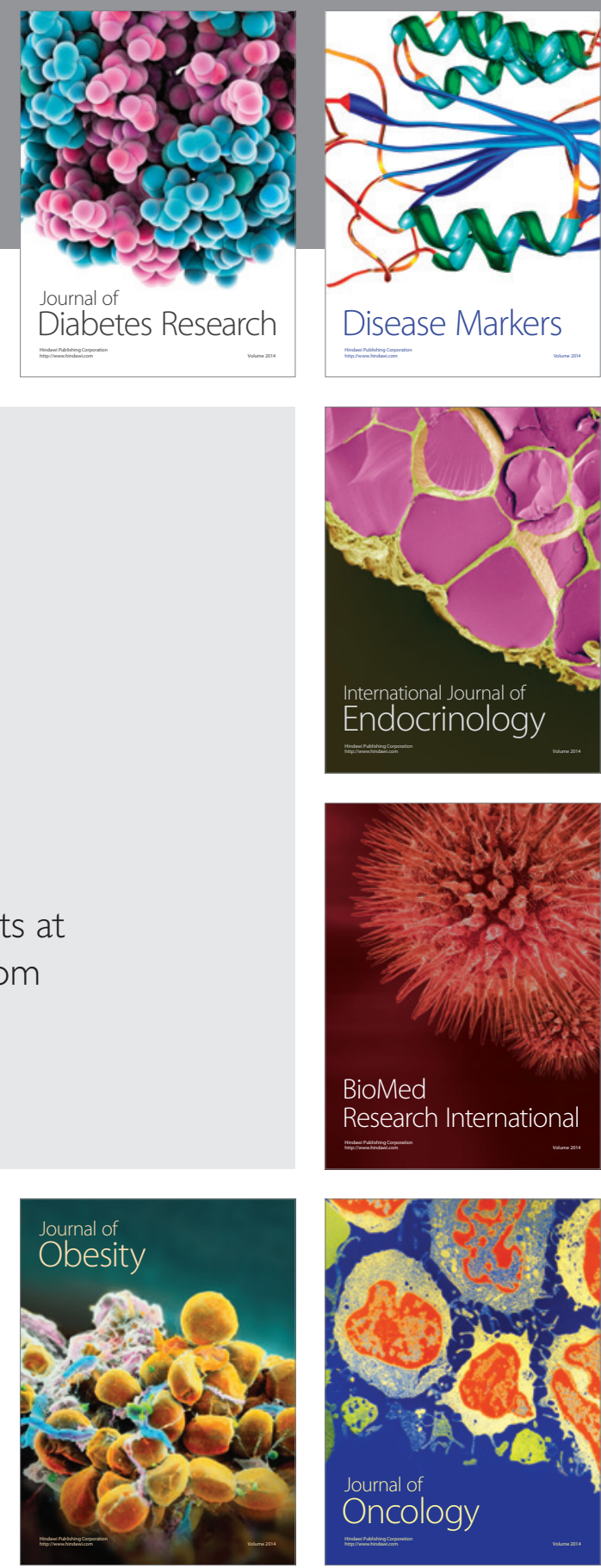

Disease Markers
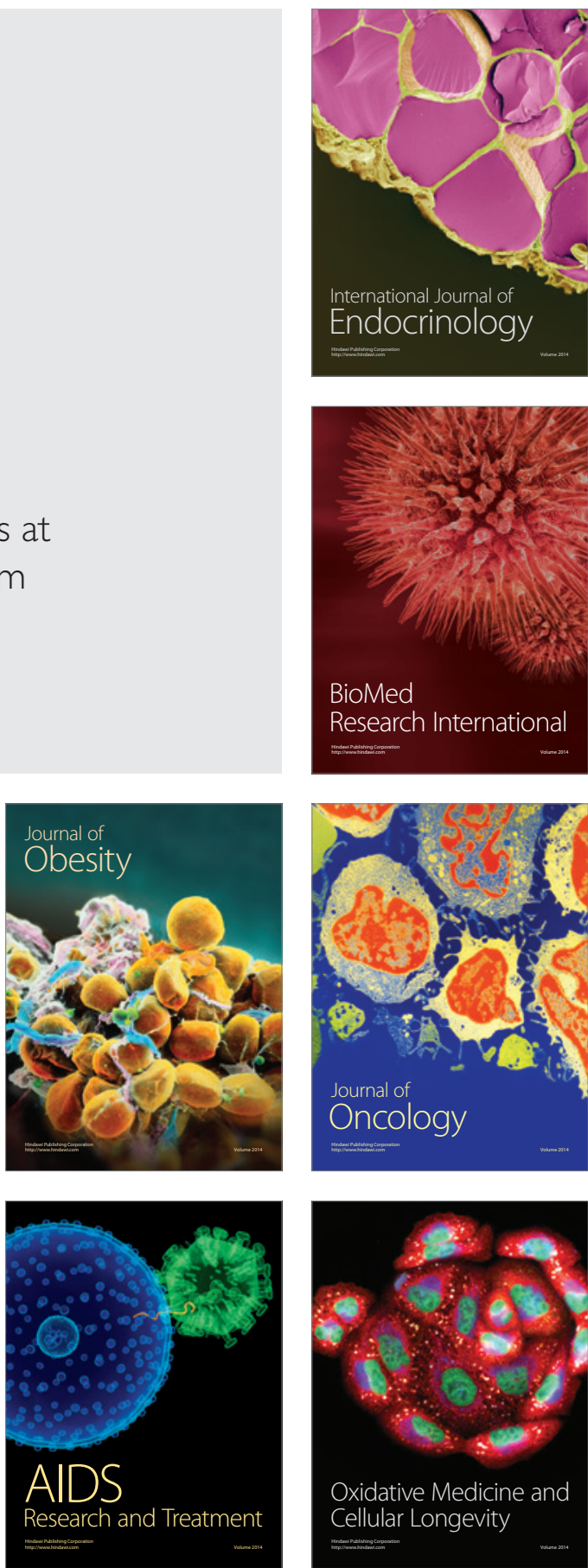